\title{
Detection and Identification of Sarcocystis cruzi (Protozoa: Apicomplexa) by Molecular and Ultrastructural Studies in Naturally Infected Korean Cattle (Bos taurus coreanae) from Daejeon, Korea
}

\author{
Tong-II Choi' ${ }^{1, \dagger}$, Eui-Ju Hong ${ }^{1, \dagger}$, Si-Yun Ryu', Cheolho Sim², Joon-Seok Chae ${ }^{3}$, Hyeon-Cheol Kim4, Jinho Park ${ }^{5}$, \\ Kyoung-Seong Choi ${ }^{6}$, Do-Hyeon $\mathrm{Yu}^{7}$, Jae-Gyu Yoo ${ }^{8}$, Bae-Keun Park ${ }^{1, *}$ \\ ${ }^{1}$ College of Veterinary Medicine, Chungnam National University, Daejeon 34134, Korea; ${ }^{2}$ Department of Biology, Baylor University, Waco, Texas \\ 76798, USA; ${ }^{2}$ Laboratory of Veterinary Internal Medicine, BK21 PLUS Program for Creative Veterinary Science Research and College of Veterinary \\ Medicine, Seoul National University, Seoul 08826, Korea; ${ }^{4}$ College of Veterinary Medicine, Kangwon National University, Chuncheon 24341, Korea; \\ ${ }^{5}$ College of Veterinary Medicine, Chonbuk National University, Jeonju 54896, Korea; ${ }^{6}$ College of Animal Science, Kyungpook National University, \\ Daegu 41566, Korea; ${ }^{7}$ College of Veterinary Medicine, Gyeongsang National University, Jinju 52828, Korea; ${ }^{8}$ Laboratory of Veterinary Clinics, \\ National Institute of Animal Science Rural Development Administration, Wanju 55365, Korea
}

\begin{abstract}
To survey the prevalence of Sarcocystis infections, 210 heart samples were collected from Korean native cattle (Bos taurus coreanae) at an abattoir in Daejeon Metropolitan City, Republic of Korea. Sarcocysts were detected form 31 specimens (14.8\%) and identified as Sarcocystis cruzi via transmission electron microscopy. The wall of S. cruzi has flattened protrusions that did not contain fibrils or microfilaments. The protrusions arose irregularly from the base, contained a fine granular substance, lacked internal microfilaments, and measured approximately 0.21-1.25 $\mu \mathrm{m}$ in length and 0.05$0.07 \mu \mathrm{m}$ in width. Sequence analysis revealed $99.5 \%$ homology to $S$. cruzi. This is the first report on the prevalence of $S$. cruzi in native cattle from the Republic of Korea.
\end{abstract}

Key words: Sarcocystis cruzi, Bos taurus coreanae, ultrastructure, 18S rRNA

\section{INTRODUCTION}

The genus Sarcocystis is related to protozoa and, as the largest genus in the phylum Apicomplexa, can cause mortality in many species of domestic and wild animals [1]; both definitive and intermediate hosts are known for only 56 species. The fine structures of sarcocyst walls may change with age and are not considered satisfactory for separating species [2]. The parasites have 2 hosts in which herbivores or omnivores act as intermediate hosts that are infected after ingesting oocysts in the feces of the definitive hosts, and carnivorous definitive hosts acquire the infection by ingesting sarcocysts in the tissue of intermediate hosts [3-5]. The cysts of Sarcocystis spp. are found worldwide in the muscles of bovid intermediate hosts. Sarco-

- Received 15 January 2018, revised 2 April 2018, accepted 5 April 2018.

*Corresponding author (bkpark@cnu.ac.kr).

†These authors contributed equally to this work.

(c) 2018, Korean Society for Parasitology and Tropical Medicine

This is an Open Access article distributed under the terms of the Creative Commons Attribution Non-Commercial License (http://creativecommons.org/licenses/by-nc/4.0) which permits unrestricted non-commercial use, distribution, and reproduction in any medium, provided the original work is properly cited. cystis infections in these hosts cause diseases including anorexia, pyremia, anemia, weight loss, abortion, and death in severe cases $[1,5,6]$.

The main objective of this study was to determine the prevalence of Sarcocystis infection in Korean native cattle. Previous Korean surveys of Sarcocystis infection among rearing cattle have indicated that higher infection rates are observed in older cattle and have identified canines as the definitive hosts, but taxonomic classification by molecular research and transmission electron microscopy (TEM) has not been achieved [7-9]. This paper describes the detection of Sarcocystis by light microscopy (LM) of heart muscle tissue sections, shows the prevalence of Sarcocystis cruzi and identifies specific species through TEM, PCR, sequencing, and phylogenetic analysis.

\section{MATERIALS AND METHODS}

Sample collection and histological processing

Total 210 cardiac muscle samples (male 3, bullock 2, and female 205) from Korean native cattle, Bos taurus coreanae, were 
obtained from November 2013 to January 2014 in a slaughterhouse located in Daejeon Metropolitan City, the Republic of Korea. Portions of each sample were fixed in 10\% neutral buffered formalin, embedded in paraffin, sectioned at $4 \mu \mathrm{m}$, and stained with hematoxylin and eosin for histological examination. The remainder of the samples were stored at $-20^{\circ} \mathrm{C}$ for PCR.

\section{TEM}

Pieces of the heart muscles were examined by LM, and those that contained cysts were fixed with $2.5 \%$ glutaraldehyde solution in $0.1 \mathrm{M}$ phosphate buffer ( $\mathrm{pH} 7.4$ ) for $4 \mathrm{hr}$. After the buffer wash, specimens were post-fixed with $1 \%$ osmium tetroxide at $4^{\circ} \mathrm{C}$ for $4 \mathrm{hr}$. Afterwards, the specimens were dehydrated in a graded ethyl alcohol series and 2 changes of propylene oxide and embedded in an epon mixture. The ultrathin sections were stained with uranyl acetate and lead citrate before examination on a Tecnai G2 TEM at $120 \mathrm{kV}$ (FEI, Hillsboro, Oregon, USA).

\section{DNA extraction for PCR}

Genomic DNA was next extracted from the frozen, cyst-positive samples that had been identified by light microscopy; the DNA was extracted using DNeasy Blood \& Tissue Kits (Qiagen, Hilden, Germany) according to the manufacturer's instruction. PCR amplification was performed using EmeraldAmp ${ }^{\circledR}$ GT PCR Master Mix (Takara, Shiga, Japan) with $1 \mu \mathrm{l}$ of the abovedescribed template gDNA. Two pairs with 18S 2L (5'-GGATA AACCGTGGTAATTCTATG-3')/3H (5'-GGCAAATGCTTTCGCAGTAG-3') and S1 (5'-GAATCCAAACCCCTTTCAGAGT$\left.3^{\prime}\right) / 1 \mathrm{H}\left(5^{\prime}\right.$-TATCCCCATCACGATGCATAC-3') primers were used to amplify the partial $18 \mathrm{~s}$ rRNA gene $[10,11]$. In order of 2L (forward) - S1 (forward) -3H (reverse)-1H (reverse) primer, they are located in Sarcocystis sequence. We expected that $2 \mathrm{~L} / 3 \mathrm{H}$ and $\mathrm{S} 1 / 1 \mathrm{H}$ primer sets make an about $850 \mathrm{bp}$ and 1,050 bp PCR products, respectively. The cycling parameter for the PCR consisted of initial denaturation at $95^{\circ} \mathrm{C}$ for 5 min followed by 40 cycles of $94^{\circ} \mathrm{C}$ for $40 \mathrm{sec}, 55^{\circ} \mathrm{C}$ for $45 \mathrm{sec}$, and $72^{\circ} \mathrm{C}$ for 1 min $30 \mathrm{sec}$, with final extension at $72^{\circ} \mathrm{C}$ for $6 \mathrm{~min}$. The PCR products were next visualized via electrophoresis on $1.2 \%$ agarose gel with a SiZer ${ }^{\mathrm{TM}}$-100 DNA marker (Intron, Seongnam, Korea) and purified using Nucleospin ${ }^{\circledR}$ Gel and PCR Clean-up (Macherey-Nagel, Düren, Germany) for sequencing.

\section{Sequencing and phylogenetic analysis}

Positive PCR products were directly sequenced by dideoxy chain termination with an automatic sequencer (ABI $3730 \mathrm{xl}$ capillary DNA sequencer, Applied Biosystems, Foster City, California, USA) using above primers and additional 17 (5'-AGAATTTCACCTCTG-3') primers. All of sequence data were linked with single sequence. The resulting sequences were aligned and then subjected to phylogenetic analyses of the SSU rRNA genes against previously sequenced Sarcocystis species as well as with other registered sequences of Sarcocysits spp. from intermediate hosts, retrieved from GenBank using the BLAST program (http://www.ncbi.nlm.nih.gov/BLAST). A score of sequences were aligned by DNASTAR (DNASTAR Inc.), Clustal W (www. clustal. org), and MEGA 6.0 [12]. Neighbor-joining $[13,14]$ methods were based on a guide tree as pairwise and multiple alignment parameters. The final alignment comprised a score of sequences with 36 taxa.

\section{RESULTS}

\section{LM findings}

Sarcocysts were found in the cardiac tissue slides of the Korean native cattle. The cysts were all in the muscle fibers. The sarcoplasms of the myocardia presented with numerous bradyzoites enclosed in the thin and smooth host cell wall. The walls of the sarcocysts were indiscernible (Fig. 1A, B). The cysts shaped oval to spherical and 37-111 ×66-304 (mean $63.1 \times 167.0) \mu \mathrm{m}$ in size. The specific species could not be accurately described by light microscopic observation on the sample slides because morphological differences could not be told from the sarcocysts of other species. Eosinophilic myositis and diffuse leukocytic infiltration were not severe.

\section{TEM findings}

The cysts were situated within the cardiac muscle fibers and the cyst walls were thin $(<1 \mu \mathrm{m}$ in width) and had hair-like, flattened protrusions. The walls consisted of 3 layers, ground substance, flattened protrusion, and sarcoplasm (Fig. 2C), and the protrusions measured approximately $0.21-1.25 \mu \mathrm{m}$ in length and $0.05-0.07 \mu \mathrm{m}$ in width, arose irregularly from the base, contained a fine granular substance, and lacked internal microfilaments (Fig. 2C, D); we observed variations in the density of the protrusions even within a given cyst. The cysts had prominent septa (Fig. 2B), and the parasites were enclosed in amorphous matrices that contained numerous oval to fusiform bradyzoites (Fig. 2A). The bradyzoites consisted of 4 regions: an anterior region that contained rhoptries and a large 

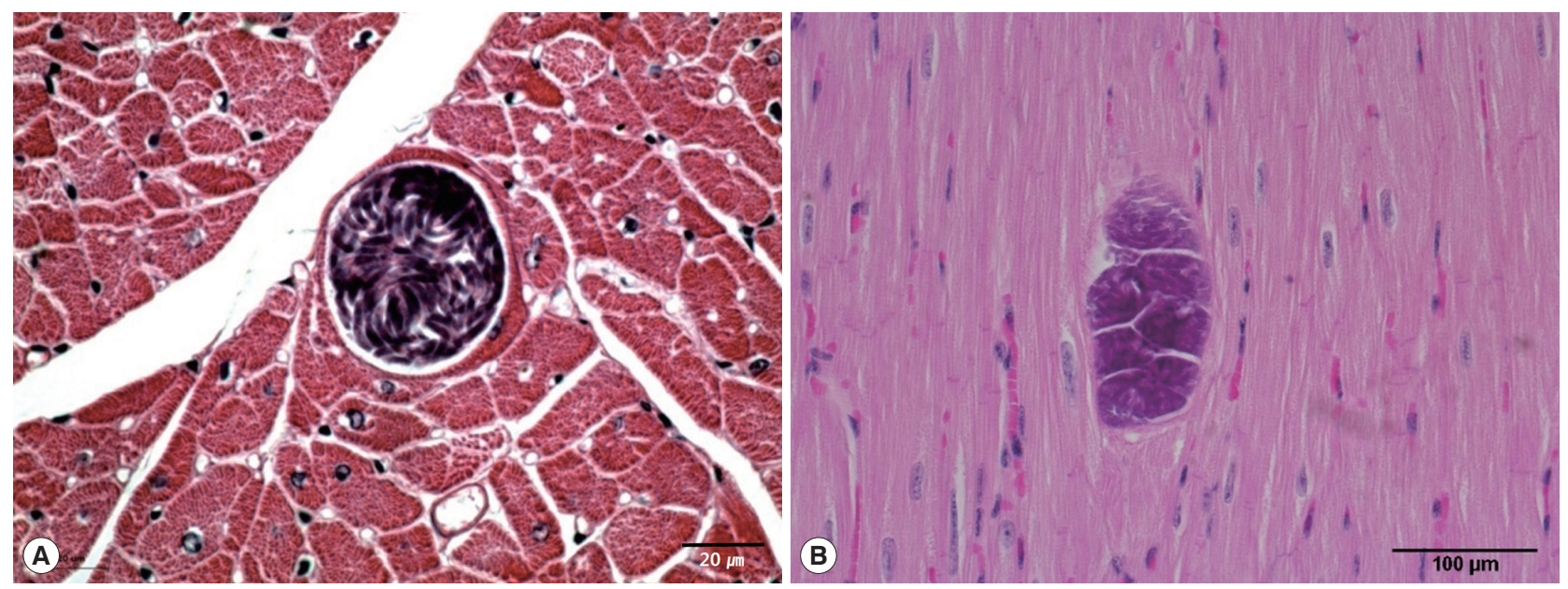

Fig. 1. Light micrographs of S. cruzi sarcocyst in the cardiac muscles of Korean native cattle. No tissue reaction. H-E stain. Scale bar=20 $\mu \mathrm{m}(\mathrm{A}), 100 \mu \mathrm{m}(\mathrm{B})$.

number of micronemes, a fourth second region with abundant amylopectin granules, a fourth third region that contained the nucleus, and a posterior region that contained abundant amylopectin granules (Fig. 2E). The multiple rhoptries appeared in the cross-sectioned anterior regions of the bradyzoites, and we counted 7 rhoptries (Fig. 2F).

\section{S rRNA sequence}

Based on the histological results, PCR amplification, sequencing, and phylogenetic analysis were performed on the $18 \mathrm{~S}$ rRNA to identify species. Positive cardiac samples were subjected to amplify $18 S$ rRNA. The PCR amplicons were approximately $1,500 \mathrm{bp}$, which was in accordance with the predicted size. Three amplicons were sequenced. BLAST searches revealed that the $18 \mathrm{~S}$ rRNA sequences showed $99.5 \%$ similarity to $S$. cruzi. GeneBank accession number with MH129611. A phylogenic tree of the 18S rRNA sequence revealed our sarcocysts branched into a group of S. cruzi from other countries (Fig. 3).

\section{DISCUSSION}

There are 3 main species of Sarcocystis in cattle: S. cruzi, of which canids are definitive hosts; $S$. hirsuta, transmitted by cats; and S. hominis, transmitted by humans. S. cruzi is the most common and important species that affects cattle [15-17]. It is one of the most common Sarcocystis spp. in bovids, with $S$. cruzi cysts most commonly found in the heart $[18,19]$. Interestingly, some species of Sarcocystis avoid certain muscle organs. For example, $S$. muris in mice, $S$. gigantea in sheep, and $S$. hirsuta in cattle do not develop in the heart [20]. It was reported that the villar protrusions in $S$. hirsuta were $8 \mu \mathrm{m}$ long and constricted at the base and they expanded laterally in the midregion and tapered distally, and the villar core contained numerous microfilaments and rows of electron-dense granules [21].

In the Republic of Korea, S. cruzi has been rarely reported [79]; however, the taxonomy has been host given, and the specific species identification through molecular work and the study of the ultrastructure have not been clearly illustrated.

In our study, LM examinations revealed 31 (14.8\%) cardiac samples that were positive for sarcocysts from a pool of 210. The sarcoplasms of the myocardia presented with bradyzoites enclosed in the thin smooth cyst walls, and the cysts ranged in morphology from oval to spherical and measured between 37 $111 \times 66-304$ (mean $63.1 \times 167.0) \mu \mathrm{m}$ in size; this depiction is similar to those in previous studies [7]. However, due to the similarity of sarcocysts in other species, including S. levinei [22], for more accurate identification, molecular work and the study of the ultrastructure are needed. The structure of the sarcocyst, particularly its walls, is characteristic of a species within a host and is commonly used in identifying Sarcocystis species; under LM, the structures of sarcocyst walls and their protrusions are decisive for assessing the species. Unfortunately, however, LM cannot discern between the 3 Sarcocystis spp. in cattle with different definitive hosts $[19,23]$. The thin-walled S. cruzi cysts can easily be distinguished from the thick-walled sarcocysts of S. hominis and $S$. hirsuta guided by tissue sections $[4,24,25] ; S$. hominis and S. hirsuta have "thick, radially striated," 2.5-9.0 $\mu \mathrm{m}$ 

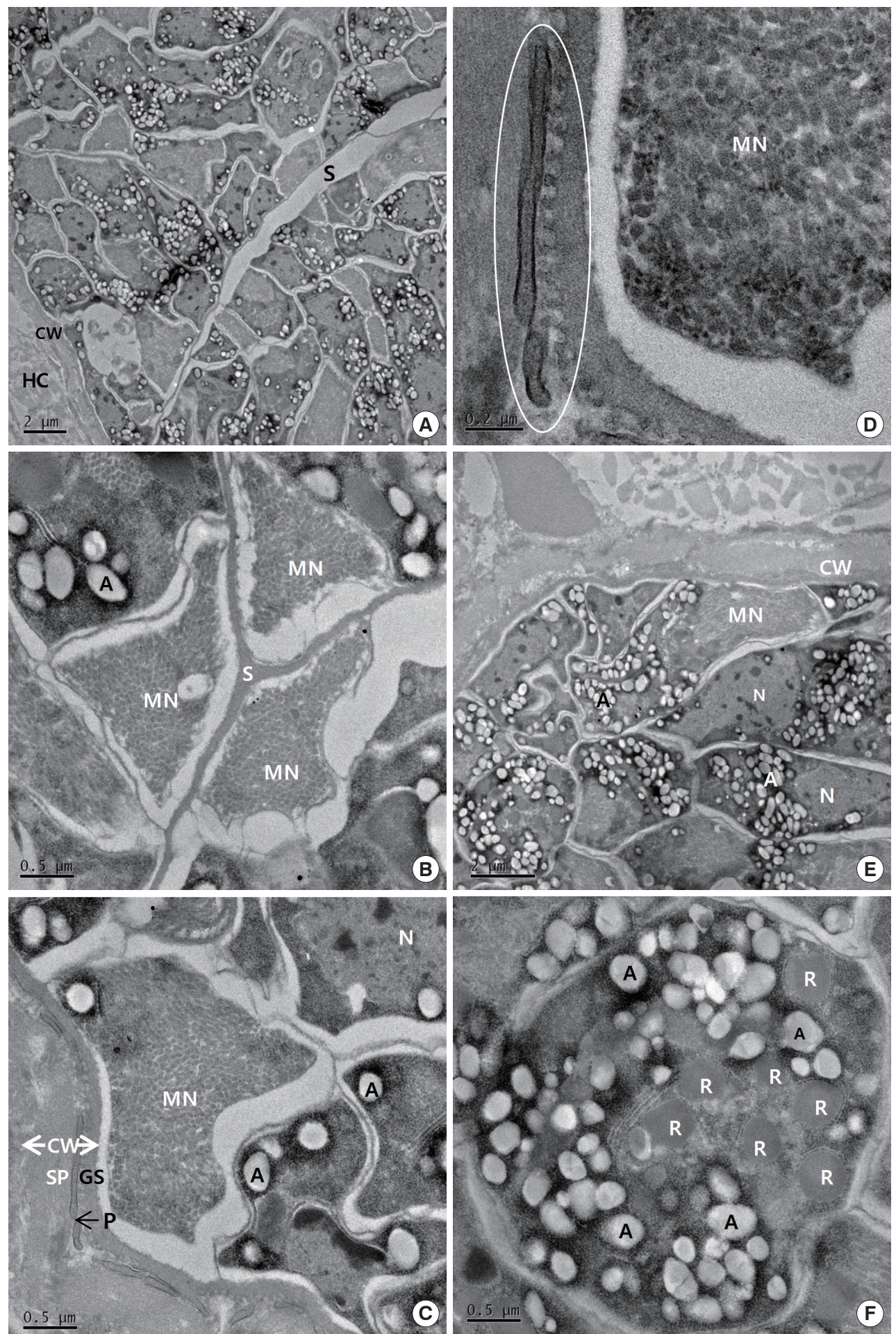

Fig. 2. Transmission electron micrographs of cyst of S. cruzi from the heart of Bos taurus coreanae. (A) A high concentration of bradyzoites is enclosed by a thin cyst wall and septa. (B) The micronemes and septa. (C) The cyst wall. Cyst wall protrusions are adjacent to the sarcoplasm of host cell. (D) The magnified protrusion (circle). Microfilaments are not found in the protrusion. (E) The micronemes and nucleus. (F) The cross sectioned anterior region of bradyzoite containing several rhoptries. In addition to several rhoptries, amylopectin granules are presented in abundance. A, amylopectin granule; CW, cyst wall; GS, ground substance; MN, micronemes; N, nucleus; P, protrusion; R, rhoptry; S, septa; SP, sarcoplasm; HC, host cell. 


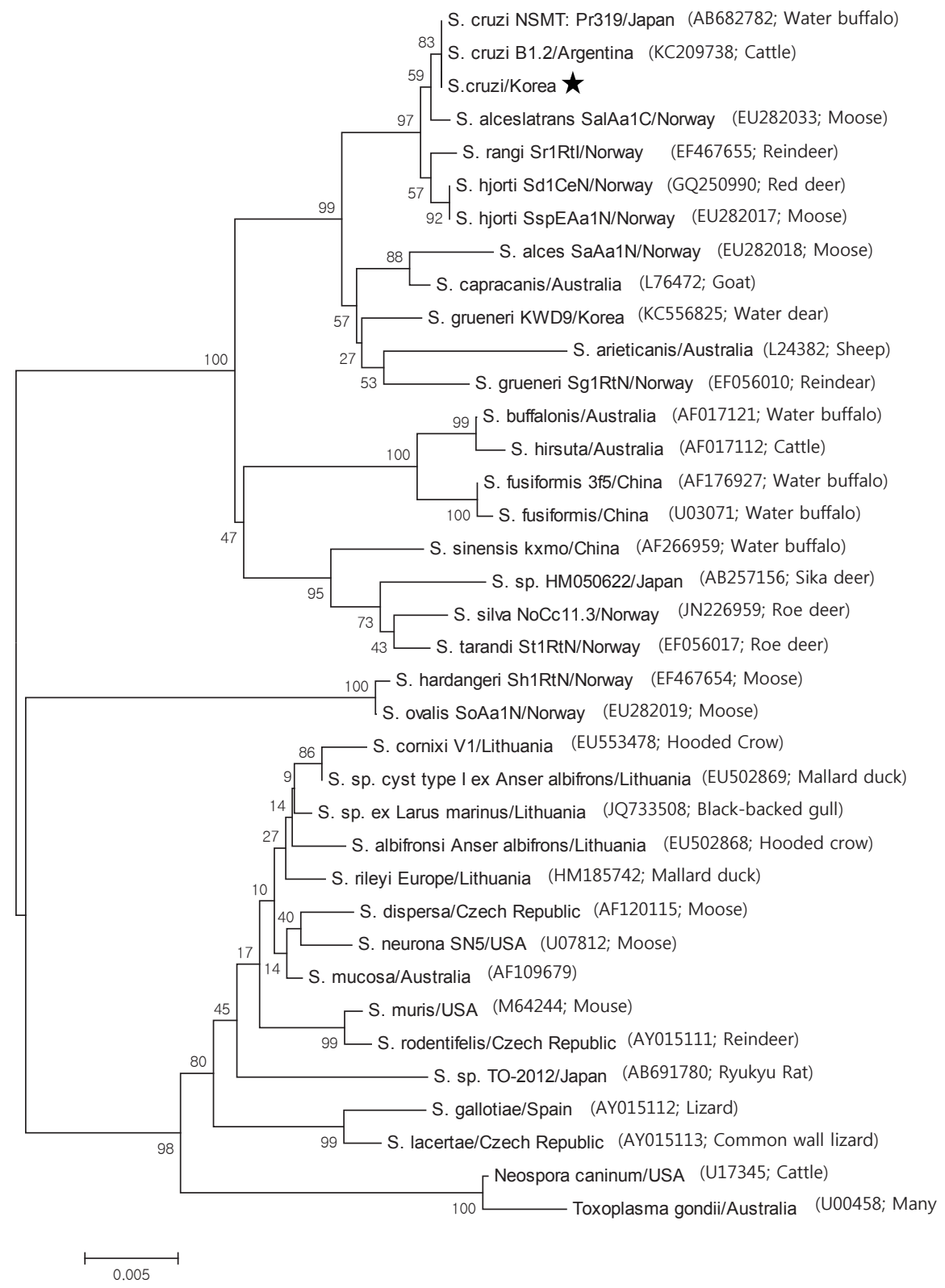

Fig. 3. Phylogenetic analysis of the $18 \mathrm{~S}$ rRNA gene sequences from Sarcocystis species and their evolutionary relationships of 36 taxa $(1.5 \mathrm{~kb})$. A star indicates that the infected samples collected from the cardiac muscles of Korean native cattle (Bos taurus coreanae) is $S$. cruzi.

cyst walls [26]. In our study, the cysts ranged in morphology from oval to spherical and measured 37-111 ×66-304 (mean $63.1 \times 167.0) \mu \mathrm{m}$ in size, and the cyst walls were indiscernible; we determined that the specific species could not be accurately described histologically. In this study, transmission emission microscopy revealed the presence of thin walls $(<1 \mu \mathrm{m}$ in width) in $S$. cruzi, but other differences in morphology could not be observed between the corresponding sarcocysts from different host species.

Under TEM, the characteristics of the cysts were similar to those described in previous studies $[26,27]$. The structure of $S$. hominis was described in the current study as having nearly perpendicular, cylindrical protrusions with many longitudinal microfilaments and few dense granules $[21,28,29]$. S. hirsute can be determined most reliably by TEM (in some cases also in semi-thin sections): the villar protrusions are club- or bulb- 
shaped in the outline, with a stalklet at the base and with microfilaments [26]. The muscular tissue cysts found in other genera (i.e., Toxoplasma gondii and Hammondia hammondi) may resemble sarcocysts. They have thin cyst walls but lack septa or a secondary cyst wall [30]. Our present study also found thin sarcocyst walls $(<1 \mu \mathrm{m}$ in width), and the septa and walls had flattened protrusions that did not contain fibrils or microfilaments.

Phylogenetic topology of 1,000 bootstraps of our samples revealed high sequence similarity to $S$. cruzi, and BLAST searches revealed that the $18 \mathrm{~S}$ rRNA sequences shared $99.5 \%$ identity with S. cruzi.

$S$. cruzi is very frequently identified in the cardiac muscles of cattle that are native to Korea. We identified the parasites as $S$. cruzi using morphological and molecular studies including light and transmission electron microscopy, PCR, and sequence analysis. This is the first report on detecting S. cruzi using TEM and $18 \mathrm{~S}$ rRNA gene sequence analysis in native cattle from the Republic of Korea.

\section{ACKNOWLEDGMENT}

This work was supported by a research fund from Chungnam National University, Daejeon, Korea.

\section{CONFLICT OF INTEREST}

The authors have no conflicts of interest concerning the work reported in this paper.

\section{REFERENCES}

1. Soulsby EJL. Helminths, Arthropods and Protozoa of Domesticated Animals. 7th ed. London, UK. Balliere Tindall. 1982, p 670-691.

2. Levine ND. The taxonomy of Sarcocystis (Protozoa, Apicomplexa) species. J Parasitol 1986; 72: 372-382.

3. Dubey JP. A review of Sarcocystis of domestic animals and of other coccidia of cats and dogs. J Am Vet Med Assoc 1976; 169: 10611078.

4. Dubey JP, Speer CA, Fayer R. Sarcocystosis of Animals and Man. Florida, USA. CAC Press Inc. 1989, p 105-145.

5. Fayer R. Production of Sarcocystis cruzi sporocysts by dogs fed experimentally infected and naturally infected beef. J Parasitol 1977; 63: 1072-1075.

6. Johnson AJ, Hildebrandt PK, Fayer R. Experimentally induced Sarcocystis infection in calves: pathology. Am J Vet Res 1975; 36:
995-999.

7. Jang H, Kang YB, Wee SH, Choi SH. Survey of Sarcocystis infections in cattle in Korea. Res Rept RDA(V) 1990; 32: 32-37 (in Korean).

8. Kang YB, Kim KS, Wee SH. A survey of Sarcocystis infections in slaughtered cattle in Korea. Korean J Vet Res 1988; 28: 387-390.

9. Wee SH, Shin SS. Experimental induction of the two-host life cycle of Sarcocystis cruzi between dogs and Korean native calves. Korean J Parasitol 2001; 39: 227-232.

10. Formisano P, Aldridge B, Alony Y, Beekhuis L, Davies E, Del Pozo J, Dunn K, English K, Morrison L, Sargison N, Seguino A, Summers BA, Wilson D, Milne E, Beard PM. Identification of Sarcocystis capracanis in cerebrospinal fluid from sheep with neurological disease. Vet Parasitol 2013; 193: 252-255.

11. Yang ZQ, Zuo YX, Yao YG, Chen XW, Yang GC, Zhang YP. Analysis of the 18S rRNA genes of Sarcocystis species suggests that the morphologically similar organisms from cattle and water buffalo should be considered the same species. Mol Biochem Parasitol 2001; 115: 283-288.

12. Tamura K, Stecher G, Peterson D, Filipski A, Kumar S. MEGA6: Molecular evolutionary genetics analysis version 6.0. Mol Biol Evol 2013; 30: 2725-2729.

13. Kimura M. A simple method for estimating evolutionary rates of base substitutions through comparative studies of nucleotide sequences. J Mol Evol 1980; 16: 111-120.

14. Saitou N, Nei M. The neighbor-joining method: a new method for reconstructing phylogenetic trees. Mol Biol Evol 1987; 4: 406425.

15. Dubey JP, Lindsay DS. Neosporosis, toxoplasmosis, and sarcocystosis in ruminants. Vet Clin North Am Food Anim Pract 2006; 22: 645-671.

16. Heydorn AO, Gestrich R, Mehlhorn H, Rommel M. Proposal for a new nomenclature of the Sarcosporidia. Z Parasitenkd 1975; 48: 73-82.

17. Mowafy NM. Sarcocystis of cattle in EL-minia, upper Egypt and ultrastructure of Sarcocystis hominis cysts. El-Minia Med Bull 2003; 14: 74-87.

18. Dubey JP, Speer CA, Epling GP. Sarcocystosis in newborn calves fed Sarcocystis cruzi sporocysts from coyotes. Am J Vet Res 1982; 43: 2147-2164.

19. Heydorn AO, Rommel M. Contributions on the life cycle of Sarcosporidia. II. Dog and cat as vectors of cattle Sarcosporidia. Berl Munch Tierarztl Wochenschr 1972; 85: 121-123 (in German).

20. Lindsay DS, Blagburn BB, Braund KG. Sarcocystis spp. and Sarcocystosis. BAM 1995; 5: 249-254.

21. Dubey JP, Speer CA, Charleston WA. Ultrastructural differentiation between sarcocysts of Sarcocystis hirsuta and Sarcocystis hominis. Vet Parasitol 1989; 34: 153-157.

22. Huong LT, Dubey JP, Uggla A. Redescription of Sarcocystis levinei Dissanaike and Kan, 1978 (Protozoa: Sarcocystidae) of the water buffalo (Bubalus bubalis). J Parasitol 1997; 83: 1148-1152.

23. Rommel M, Heydorn AO, Gruber F. Life cycle of Sarcosporidia. 1. The sporocyst of $S$. tenella in cat feces. Berl Munch Tierarztl Woch- 
enschr 1972; 85: 101-105 (in German).

24. Berhgmann V, Kinder E. Ultrastruktur der Zystenwand von Sarkozysten in Muskelfasern spontan infizierter Rinder. Monatsh Veterinärmed 1975; 30: 945-947 (in German).

25. Vercruysse J, Fransen J, van Goubergen M. The prevalence and identity of Sarcocystis cysts in cattle in Belgium. Zentralbl Veterinarmed B 1989; 36: 148-153.

26. Odening K, Wesemeier HH, Walter G, Bockhardt I. On the morphological diagnostics and host specificity of the Sarcocystis species of some domesticated and wild bovini (cattle, banteng and bison). Appl Parasitol 1995; 36: 161-178.

27. Xiang Z, He Y, Zhao H, Rosenthal BM, Dunams DB, Li X, Zuo Y,
Feng G, Cui L, Yang Z. Sarcocystis cruzi: comparative studies confirm natural infections of buffaloes. Exp Parasitol 2011; 127: 460466.

28. Moré G, Abrahamovich P, Jurado S, Bacigalupe D, Marin JC, Rambeaud M, Venturini L, Venturini MC. Prevalence of Sarcocystis spp. in Argentinean cattle. Vet Parasitol 2011; 177: 162-165.

29. Saito M, Shibata Y, Kubo M, Sakakibara I, Yamada A, Itagaki H. First isolation of Sarcocystis hominis from cattle in Japan. J Vet Med Sci 1999; 61: 307-309.

30. Mehlhorn H, Frenkel JK. Ultrastructural comparison of cysts and zoites of Toxoplasma gondii, Sarcocystis muris, and Hammondia hammondi in skeletal muscle of mice. J Parasitol 1980; 66: 59-67. 
